\title{
Eigenvectors in the superintegrable model II: ground-state sector
}

\author{
Helen Au-Yang ${ }^{1,2}$ and Jacques H H Perk ${ }^{1,2}$ \\ ${ }^{1}$ Department of Physics, Oklahoma State University, 145 Physical Sciences, Stillwater, \\ OK 74078-3072, USA \\ 2 Centre for Mathematics and its Applications \& Department of Theoretical Physics, \\ Australian National University, Canberra, ACT 2600, Australia \\ E-mail: helenperk@yahoo.com and perk@okstate.edu
}

Received 18 June 2009, in final form 19 July 2009

Published 28 August 2009

Online at stacks.iop.org/JPhysA/42/375208

\begin{abstract}
In 1993, Baxter gave $2^{m_{Q}}$ eigenvalues of the transfer matrix of the $N$-state superintegrable chiral Potts model with the spin-translation quantum number $Q$, where $m_{Q}=\lfloor(N L-L-Q) / N\rfloor$. In our previous paper we studied the $Q=0$ ground-state sector, when the size $L$ of the transfer matrix is chosen to be a multiple of $N$. It was shown that the corresponding $\tau_{2}$ matrix has a degenerate eigenspace generated by the generators of $r=m_{0}$ simple $\mathfrak{s l}_{2}$ algebras. These results enable us to express the transfer matrix in the subspace in terms of these generators $\mathbf{E}_{m}^{ \pm}$and $\mathbf{H}_{m}$ for $m=1, \ldots, r$. Moreover, the corresponding $2^{r}$ eigenvectors of the transfer matrix are expressed in terms of rotated eigenvectors of $\mathbf{H}_{m}$.
\end{abstract}

PACS numbers: $02.20 . \mathrm{Uw}, 05.50 .+\mathrm{q}$, 64.60.De, 75.10.Hk, 75.10.Jm

In our previous paper [1], we have shown that to calculate the correlation functions of the chiral Potts model, one only needs to study the eigenvectors of the superintegrable model. Since the transfer matrix and the matrix $\tau_{2}\left(t_{q}\right)$ have the same eigenvectors, we have examined the eigenspace of $\tau_{2}\left(t_{q}\right)$ which has degeneracy $2^{r}$, where $r=L(N-1) / N$ with $L$-the length of the transfer matrix-chosen to be a multiple of $N$. This space is a highest-weight representation of an $\mathcal{L}\left(\mathfrak{s l}_{2}\right)$ loop algebra which turns out to be a direct sum of $r$ copies of the simple algebra $\mathfrak{s l}_{2}$ whose generators are $\mathbf{E}_{m}^{ \pm}$and $\mathbf{H}_{m}$ for $m=1, \ldots, r$.

In 1989, Baxter in his paper on the 'Superintegrable Chiral Potts Model' [2] suggested that the transfer matrix restricted to a certain subspace may be written as

$$
\mathcal{T}_{Q}=C_{Q} \rho^{L} \prod_{j=1}^{r}\left\{G I+\left(1-k^{\prime} \cos \theta_{j}\right) \sigma_{j}^{z}-k^{\prime} \sin \theta_{j} \sigma_{j}^{x}\right\}
$$


(see equation (2.16) of [2]). Our attempt is to do exactly that. However, as the modulus $k^{\prime}$ in that paper [2] is different from the usual $k^{\prime}$ of other papers, we shall consider the transfer matrix of his later papers $[3,4]$.

From equation (6.2) of $[3]^{3}$ the transfer matrices of the superintegrable model can be written as

$T_{q}=N^{\frac{1}{2} L} \frac{\left(x_{q}-y_{p}\right)^{L}}{\left(x_{q}^{N}-y_{p}^{N}\right)^{L}} \mathcal{T}\left(x_{q}, y_{q}\right), \quad \hat{T}_{q}=N^{\frac{1}{2} L} \frac{\left(x_{q}-x_{p}\right)^{L}}{\left(x_{q}^{N}-x_{p}^{N}\right)^{L}} \hat{\mathcal{T}}\left(x_{q}, y_{q}\right)$.

Baxter showed that the eigenvalues of $\mathcal{T}\left(x_{q}, y_{q}\right)$ for spin shift quantum number $Q=0$, or equivalently $\mathcal{T}_{0}\left(x_{q}, y_{q}\right)$ corresponding to $(\mathrm{I} .10)^{4}$, depend on $\lambda_{q}=\mu_{q}^{N}$ only and are denoted by $\mathcal{G}\left(\lambda_{q}\right)$. The corresponding eigenvalue $\hat{\mathcal{G}}\left(\lambda_{q}\right)$ of $\hat{\mathcal{T}}_{0}\left(x_{q}, y_{q}\right)$ can be made equal to $\mathcal{G}\left(\lambda_{q}\right)$ by simple rescaling of the eigenvectors $\mathbf{x}$ and $\mathbf{y}$, so that

$\mathcal{T}_{0}\left(x_{q}, y_{q}\right) \mathbf{x}=\mathcal{G}\left(\lambda_{q}\right) \mathbf{y}, \quad \hat{\mathcal{T}}_{0}\left(x_{q}, y_{q}\right) \mathbf{y}=\mathcal{G}\left(\lambda_{q}\right) \mathbf{x}, \quad \hat{\mathcal{T}}_{0}\left(y_{q}, x_{q}\right) \mathbf{y}=\mathcal{G}\left(\lambda_{q}^{-1}\right) \mathbf{x}$.

Thus, (I.9) and (6.24) of [3] become ${ }^{5}$

$$
\mathcal{G}\left(\lambda_{q}\right) \mathcal{G}\left(\lambda_{q}^{-1}\right)=t_{p}^{r N} N \mathcal{P}\left(t_{q} / t_{p}\right)=t_{p}^{r N} N \prod_{j=1}^{r}\left[\left(t_{q} / t_{p}\right)^{N}-z_{j}\right]
$$

where $z_{j}$ and $z_{j}^{-1}$ are the roots of the Drinfeld polynomial $P(z)=P\left(t^{N}\right)=\mathcal{P}(t)$, see (I.11) with $Q=0$ and (I.41). From (I.2) we have

$$
k^{2} t_{q}^{N}=1+k^{\prime 2}-k^{\prime}\left(\lambda_{q}+\lambda_{q}^{-1}\right)
$$

Substituting this equation into (4), and letting

$$
2 \cosh 2 \theta_{j}=k^{\prime}+k^{\prime-1}-k^{2} t_{p}^{N} z_{j} / k^{\prime}
$$

we find

$$
\mathcal{G}\left(\lambda_{q}\right) \mathcal{G}\left(\lambda_{q}^{-1}\right)=N\left(\frac{k^{\prime}}{k^{2}}\right)^{r} \prod_{j=1}^{r} \mathrm{e}^{\mp 2 \theta_{j}}\left(\mathrm{e}^{ \pm 2 \theta_{j}}-\lambda_{q}^{-1}\right)\left(\mathrm{e}^{ \pm 2 \theta_{j}}-\lambda_{q}\right) .
$$

Therefore, as $\mathcal{G}\left(\lambda_{q}\right)$ is a polynomial in $\lambda_{q}^{-1}$ according to section 6 of [3], the $2^{r}$ implied eigenvalues of the transfer matrix $\mathcal{T}_{0}\left(x_{q}, y_{q}\right)$ in (I.10) are

$\mathcal{G}\left(\lambda_{q}\right)=\rho^{r} \prod_{j=1}^{r}\left[\cosh \theta_{j}\left(1-\lambda_{q}^{-1}\right) \pm \sinh \theta_{j}\left(1+\lambda_{q}^{-1}\right)\right]=\prod_{j=1}^{r}\left(A_{j} \pm B_{j}\right)$,

$A_{j}=\rho \cosh \theta_{j}\left(1-\lambda_{q}^{-1}\right), \quad B_{j}=\rho \sinh \theta_{j}\left(1+\lambda_{q}^{-1}\right), \quad \rho^{r}=N^{\frac{1}{2}}\left(k^{\prime} / k^{2}\right)^{\frac{1}{2} r}$,

cf (20) of [4]. These are the results of Baxter, written in different forms.

We shall now attempt to express the transfer matrix $\mathcal{T}_{0}\left(x_{q}, y_{q}\right)$ of (I.10) for $Q=0$ in terms of the generators $\mathbf{E}_{m}^{ \pm}$and $\mathbf{H}_{m}$, defined for $m=1, \ldots, r$ in [1], and also obtain the corresponding eigenvectors.

Similar to what Davies [5] did, we define the polynomials

$$
f_{j}(z)=\prod_{\ell \neq j} \frac{z-z_{\ell}}{z_{j}-z_{\ell}}=\sum_{n=0}^{r-1} \beta_{j, n} z^{n}, \quad f_{j}\left(z_{k}\right)=\delta_{j, k},
$$

3 This simplifies under the periodic boundary condition, i.e. $r=m_{P}=0$ in [3] with $r$ being Baxter's skewness parameter here, not to be confused with $r=L(N-1) / N$ in this paper.

4 All equations in [1] are denoted here by prefacing $I$ to its equation number.

5 Equation (I.11) with $Q=0, t \equiv t_{q} / t_{p}$ and (6.25) of [3] with $P_{a}=P_{b}=0, F\left(t_{q}\right) \equiv 1$ differ by a factor $N$. 
where $\beta_{j, n}$ are the elements of the inverse of the Vandermonde matrix [6], such that

$\sum_{n=0}^{r-1} \beta_{j, n} z_{k}^{n}=\delta_{j, k}, \quad \sum_{k=1}^{r} z_{k}^{n} \beta_{k, m}=\delta_{n, m} \quad$ for $\quad 0 \leqslant n \leqslant r-1$.

Consequently, (I.45) ${ }^{6}$ rewritten here as

$\mathbf{x}_{n}^{\mp}= \pm \sum_{m=1}^{r} z_{m}^{-n} \mathbf{E}_{m}^{ \pm}= \pm \sum_{m=1}^{r} z_{m^{*}}^{n} \mathbf{E}_{m}^{ \pm}, \quad \mathbf{h}_{n}=\sum_{m=1}^{r} z_{m}^{-n} \mathbf{H}_{m}=\sum_{m=1}^{r} z_{m^{*}}^{n} \mathbf{H}_{m}$,

where $n \in \mathbb{Z}$ and $m^{*}$ is the subscript for which $z_{m^{*}}=1 / z_{m}$, can be inverted as

$$
\mathbf{E}_{m}^{ \pm}= \pm \sum_{n=0}^{r-1} \beta_{m^{*}, n} z_{m}^{\ell} \mathbf{x}_{n+\ell}^{\mp}, \quad \mathbf{H}_{m}=\sum_{n=0}^{r-1} \beta_{m^{*}, n} z_{m}^{\ell} \mathbf{h}_{n+\ell},
$$

valid for $m=1, \ldots, r$ and $\ell \in \mathbb{Z}$.

In appendix $A$, we shall show that

$$
\mathbf{H}_{m}|\Omega\rangle=-|\Omega\rangle, \quad\left(\mathbf{E}_{m}^{+}\right)^{2}|\Omega\rangle=0,
$$

with $|\Omega\rangle$ the 'ferromagnetic' ground state of [1], whereas in appendix B we shall give some further results for $\mathbf{E}_{j}^{+} \mathbf{E}_{m}^{+}|\Omega\rangle$.

Deguchi and Nishino have studied a similar loop algebra [7-10]. They have shown that there exists a transformation to map the generators of the two loop algebras [10]. The proofs of the identities for the related operators are also given in [9]. (It can be verified that our $\mathbf{E}_{j}^{ \pm}$ is proportional to some $\rho_{j}^{\mp}(\mathbf{a} ; s)$ of [9], noting that Deguchi essentially uses Prony's method [6] to invert the Vandermonde matrix, but leaves out the denominators in [6].)

From the commutation relations,

$\left[\mathbf{E}_{m}^{+}, \mathbf{E}_{n}^{-}\right]=\delta_{m, n} \mathbf{H}_{m}, \quad\left[\mathbf{H}_{m}, \mathbf{E}_{n}^{ \pm}\right]= \pm 2 \delta_{m, n} \mathbf{E}_{m}^{ \pm}, \quad\left[\mathbf{E}_{m}^{+}, \mathbf{E}_{n}^{+}\right]=\left[\mathbf{E}_{m}^{-}, \mathbf{E}_{n}^{-}\right]=0$,

cf (I.46), we find

$$
\mathbf{H}_{m} \mathbf{E}_{n}^{+}|\Omega\rangle=-(-1)^{\delta_{m, n}} \mathbf{E}_{n}^{+}|\Omega\rangle .
$$

Thus by operating with various products given by the subsets of $\mathbf{E}_{1}^{+}, \mathbf{E}_{2}^{+}, \ldots, \mathbf{E}_{r}^{+}$on $|\Omega\rangle$, we obtain the eigenvectors of $\mathbf{H}_{m}$, with eigenvalues $\xi_{m}$ and $\xi_{m}= \pm 1$ depending on whether $m$ is in the subset or not. Following the example of Onsager [11], the $2^{r}$ eigenvectors are denoted by the eigenvalues $\xi_{m}$, namely,

$$
\Psi\left(\xi_{1}, \xi_{2}, \ldots, \xi_{r}\right)=\prod_{m \in J_{n}} \mathbf{E}_{m}^{+}|\Omega\rangle, \quad \xi_{j}= \pm 1
$$

where $J_{n}=\left(j_{1}, \ldots, j_{n}\right)$ is any subset of $(1,2, \ldots, r)$ for $n=0, \ldots, r$, with $\xi_{j}=1$ for $j \in J_{n}$, and $\xi_{j}=-1$ for $j \notin J_{n}$. Equivalently,

$$
\mathbf{H}_{j} \Psi\left(\xi_{1}, \xi_{2}, \ldots, \xi_{r}\right)=\xi_{j} \Psi\left(\xi_{1}, \xi_{2}, \ldots, \xi_{r}\right) .
$$

Particularly, for $n=0$ and $n=r$, we have

$$
|\Omega\rangle=\Psi(-1,-1, \ldots,-1), \quad|\bar{\Omega}\rangle=\Psi(1,1, \ldots, 1) .
$$

6 We have replaced $\mathbf{E}_{m}^{\mp} \rightarrow \pm \mathbf{E}_{m}^{ \pm}$in (I.45), so that we obtain the more usual (15) rather than (I.46). Also, $z_{m}$ and $1 / z_{m}$ are both zeros of $P(z)$ when $Q=0$, because of the symmetry of the coefficients of the polynomial $P(z)$ in (I.41), so that (12) and (I.45) are equivalent. The changes made seem to be appropriate for the $Q \neq 0$ case. 
Because $\xi_{j}= \pm 1$, it is easy to see that $\mathbf{H}_{j}^{2}=\mathbf{1}$ in this restricted eigenspace. Since $\mathbf{E}_{j}^{-}|\Omega\rangle=0$ and $\mathbf{H}_{j}|\Omega\rangle=-|\Omega\rangle$, we find from (15)

$$
\begin{aligned}
\mathbf{E}_{j}^{-} \Psi\left(\xi_{1}, \xi_{2}, \ldots, \xi_{r}\right) & =\mathbf{E}_{j}^{-} \prod_{m \in J_{n}} \mathbf{E}_{m}^{+}|\Omega\rangle \\
& = \begin{cases}\Psi\left(\xi_{1}, \ldots, \xi_{j-1},-\xi_{j}, \xi_{j+1}, \ldots, \xi_{r}\right) & \text { if } j \in J_{n}, \\
0 & \text { if } j \notin J_{n} .\end{cases}
\end{aligned}
$$

Likewise, using $\left(\mathbf{E}_{j}^{+}\right)^{2}|\Omega\rangle=0$ we obtain

$$
\begin{aligned}
\mathbf{E}_{j}^{+} \Psi\left(\xi_{1}, \xi_{2}, \ldots, \xi_{r}\right) & =\mathbf{E}_{j}^{+} \prod_{m \in J_{n}} \mathbf{E}_{m}^{+}|\Omega\rangle \\
& = \begin{cases}0 & \text { if } j \in J_{n}, \\
\Psi\left(\xi_{1}, \ldots, \xi_{j-1},-\xi_{j}, \xi_{j+1}, \ldots, \xi_{r}\right) & \text { if } j \notin J_{n} .\end{cases}
\end{aligned}
$$

Consequently, we find

$$
\left(\mathbf{E}_{j}^{+}+\mathbf{E}_{j}^{-}\right) \Psi\left(\xi_{1}, \xi_{2}, \ldots, \xi_{r}\right)=\Psi\left(\xi_{1}, \ldots, \xi_{j-1},-\xi_{j}, \xi_{j+1}, \ldots, \xi_{r}\right)
$$

and

$$
\left(\mathbf{E}_{j}^{+}+\mathbf{E}_{j}^{-}\right)^{2} \Psi\left(\xi_{1}, \xi_{2}, \ldots, \xi_{r}\right)=\Psi\left(\xi_{1}, \ldots, \xi_{j}, \ldots, \xi_{r}\right)
$$

From the commutation relation (15), we can show

$$
\begin{array}{ll}
{\left[\mathbf{H}_{j},\left(\mathbf{E}_{j}^{+}+\mathbf{E}_{j}^{-}\right)\right]=2\left(\mathbf{E}_{j}^{+}-\mathbf{E}_{j}^{-}\right),} & {\left[\mathbf{H}_{j},\left(\mathbf{E}_{j}^{+}-\mathbf{E}_{j}^{-}\right)\right]=2\left(\mathbf{E}_{j}^{+}+\mathbf{E}_{j}^{-}\right),} \\
{\left[\left(\mathbf{E}_{j}^{+}-\mathbf{E}_{j}^{-}\right),\left(\mathbf{E}_{j}^{+}+\mathbf{E}_{j}^{-}\right)\right]=2 \mathbf{H}_{j},} & \left(\mathbf{E}_{j}^{+}-\mathbf{E}_{j}^{-}\right)^{2}=-1 .
\end{array}
$$

Now, similar to what Onsager did for the Ising model in [11], we express the transfer matrix $\mathcal{T}_{0}\left(x_{q}, y_{q}\right)$ in (I.10) within the eigenspace of the eigenvalues given in (8) as

$$
\begin{aligned}
& \mathcal{T}_{0}\left(x_{q}, y_{q}\right)=\mathcal{S} \prod_{j=1}^{r}\left(A_{j}-\mathbf{H}_{j} B_{j}\right) \mathcal{R}^{-1}, \\
& \hat{\mathcal{T}}_{0}\left(x_{q}, y_{q}\right)=\mathcal{R} \prod_{j=1}^{r}\left(A_{j}-\mathbf{H}_{j} B_{j}\right) \mathcal{S}^{-1}, \quad \hat{\mathcal{T}}_{0}\left(y_{q}, x_{q}\right)=\mathcal{R} \prod_{j=1}^{r}\left(\bar{A}_{j}-\mathbf{H}_{j} \bar{B}_{j}\right) \mathcal{S}^{-1},
\end{aligned}
$$

where $\mathcal{S}$ and $\mathcal{R}$ are some complex rotation operators, which shall be determined, and $\bar{A}_{j}$ and $\bar{B}_{j}$ in (27) can be obtained from $A_{j}$ and $B_{j}$ in (9) by letting $\lambda_{q}^{-1} \rightarrow \lambda_{q}$.

If we introduce

$$
\left|\mathcal{X}_{i}\right\rangle=\mathcal{R} \Psi\left(\xi_{1}, \xi_{2}, \ldots, \xi_{r}\right), \quad\left|\mathcal{Y}_{i}\right\rangle=\mathcal{S} \Psi\left(\xi_{1}, \xi_{2}, \ldots, \xi_{r}\right)
$$

for $i=1, \ldots, 2^{r}$, then we find from (18) and (26), cf also (3), that

$\mathcal{T}_{0}\left(x_{q}, y_{q}\right)\left|\mathcal{X}_{i}\right\rangle=\mathcal{G}\left(\lambda_{q},\left\{\xi_{j}\right\}\right)\left|\mathcal{Y}_{i}\right\rangle, \quad \hat{\mathcal{T}}_{0}\left(x_{q}, y_{q}\right)\left|\mathcal{Y}_{i}\right\rangle=\mathcal{G}\left(\lambda_{q},\left\{\xi_{j}\right\}\right)\left|\mathcal{X}_{i}\right\rangle$,

where the eigenvalues are

$$
\mathcal{G}\left(\lambda_{q},\left\{\xi_{j}\right\}\right)=\prod_{j=1}^{r}\left(A_{j}-\xi_{j} B_{j}\right) .
$$

Particularly, the 'largest' eigenvalue is

$$
\mathcal{G}_{0}\left(\lambda_{q}\right)=\mathcal{G}\left(\lambda_{q},\{-1\}\right)=\prod_{j=1}^{r}\left(A_{j}+B_{j}\right),
$$


with the ground-state eigenvector

$$
\left|\mathcal{X}_{1}\right\rangle=\mathcal{R} \Psi(-1,-1, \ldots,-1)=\mathcal{R}|\Omega\rangle .
$$

The rotations $\mathcal{R}$ and $\mathcal{S}$ should be chosen such that they are independent of $q$ as the transfer matrices with different $q$ commute. They are determined from the explicit form of the transfer matrices given in (2). We now calculate the matrix elements of the transfer matrices. Using (I.4) and (I.10), we rewrite the transfer matrices in (2) (from configuration $\{\sigma\}$ to $\left\{\sigma^{\prime}\right\}$ in the row above) in terms of the edge variables $\left\{n_{i}\right\}$ and $\left\{n_{i}^{\prime}\right\}$ with $n_{i}=\sigma_{i}-\sigma_{i+1}$ as

$$
\begin{aligned}
& \left\langle\left\{n_{i}^{\prime}\right\}\left|\mathcal{T}_{Q}\left(x_{q}, y_{q}\right)\right|\left\{n_{i}\right\}\right\rangle=N^{-\frac{1}{2} L} \sum_{a=0}^{N-1} \omega^{-Q a} \prod_{j=1}^{L}\left[\left(y_{p}^{N}-x_{q}^{N}\right)\left(y_{p}-x_{q}\right)^{-1}\right. \\
& \left.\times W_{p q}\left(a-N_{j}+N_{j}^{\prime}\right) \bar{W}_{p^{\prime} q}\left(a-N_{j+1}+N_{j}^{\prime}\right)\right], \quad N_{j}=\sum_{\ell<j} n_{\ell}=\sigma_{1}-\sigma_{j},
\end{aligned}
$$

where $a=\sigma_{1}-\sigma_{1}^{\prime}$, and

$W_{p q}(n)=\left(\frac{\mu_{p}}{\mu_{q}}\right)^{n} \prod_{j=1}^{n} \frac{y_{q}-x_{p} \omega^{j}}{y_{p}-x_{q} \omega^{j}}, \quad \bar{W}_{p^{\prime} q}(n)=\left(\frac{\mu_{q}}{\mu_{p}}\right)^{n} \prod_{j=1}^{n} \frac{\omega y_{p}-x_{q} \omega^{j}}{y_{q}-x_{p} \omega^{j}}$.

Canceling out the common factors in the weights, (33) becomes

$$
\begin{aligned}
\left\langle\left\{n_{i}^{\prime}\right\}\left|\mathcal{T}_{Q}\left(x_{q}, y_{q}\right)\right|\left\{n_{i}\right\}\right\rangle=N^{-\frac{1}{2} L} \sum_{a=0}^{N-1} \omega^{-Q a} & \\
\times & \prod_{j=1}^{L}\left[\frac{\left(y_{p}^{N}-x_{q}^{N}\right) \omega^{a-N_{j+1}+N_{j}^{\prime}}}{y_{p}-x_{q} \omega^{a-N_{j+1}+N_{j}^{\prime}}}\left(\frac{\mu_{p}}{\mu_{q}}\right)^{n_{j}} \prod_{\ell=1}^{n_{j}} \frac{y_{q}-x_{p} \omega^{\ell+a-N_{j+1}+N_{j}^{\prime}}}{y_{p}-x_{q} \omega^{\ell+a-N_{j+1}+N_{j}^{\prime}}}\right] .
\end{aligned}
$$

It is easy to see that for the two ground states,

$$
|\Omega\rangle \leftrightarrow n_{i} \equiv N_{i} \equiv 0 \quad \text { or } \quad|\bar{\Omega}\rangle \leftrightarrow n_{i} \equiv N-1, \quad N_{i} \equiv(i-1)(N-1),
$$

the above expression simplifies to

$$
\left\langle\left\{n_{i}\right\}\left|\mathcal{T}_{Q}\left(x_{q}, y_{q}\right)\right| \Omega\right\rangle=N^{-\frac{1}{2} L} \sum_{a=0}^{N-1} \omega^{-Q a} \prod_{j=1}^{L} \frac{\omega^{a+N_{j}}\left(y_{p}^{N}-x_{q}^{N}\right)}{y_{p}-x_{q} \omega^{a+N_{j}}},
$$

and

$$
\left\langle\left\{n_{i}\right\}\left|\mathcal{T}_{Q}\left(x_{q}, y_{q}\right)\right| \bar{\Omega}\right\rangle=N^{-\frac{1}{2} L} \sum_{a=0}^{N-1} \omega^{-Q a} \prod_{j=1}^{L}\left(\frac{\mu_{p}}{\mu_{q}}\right)^{N-1} \frac{\omega^{a+j+N_{j}}\left(y_{q}^{N}-x_{p}^{N}\right)}{y_{q}-x_{p} \omega^{a+j+N_{j}}},
$$

where also $n_{1}+\cdots+n_{L} \equiv 0(\bmod N)$, as is required by the periodic boundary conditions. For (38) we have used $\prod_{\ell=1}^{N}\left(y-x \omega^{\ell}\right)=y^{N}-x^{N}$. Particularly, when $n_{1}=\cdots=n_{L}=0$, we find $N_{j}=0$ and (37) becomes

$$
\left\langle\Omega\left|\mathcal{T}_{Q}\left(x_{q}, y_{q}\right)\right| \Omega\right\rangle=N^{1-\frac{1}{2} L} y_{p}^{r N}\left(x_{q} / y_{p}\right)^{Q} \mathcal{P}_{Q}\left(x_{q} / y_{p}\right),
$$

while for $n_{1}=\cdots=n_{L}=N-1$, we substitute $N_{j}=(j-1)(N-1)$ into (37) to obtain

$$
\left\langle\bar{\Omega}\left|\mathcal{T}_{Q}\left(x_{q}, y_{q}\right)\right| \Omega\right\rangle=N^{1-\frac{1}{2} L} \delta_{Q, 0} \omega^{-L(L+1) / 2}\left(y_{p}^{N}-x_{q}^{N}\right)^{r} .
$$

Similarly we find from (38)

$$
\left\langle\bar{\Omega}\left|\mathcal{T}_{Q}\left(x_{q}, y_{q}\right)\right| \bar{\Omega}\right\rangle=N^{1-\frac{1}{2} L} \omega^{Q}\left(\mu_{p} y_{q} / \mu_{q}\right)^{r N}\left(x_{p} / y_{q}\right){ }^{Q} \mathcal{P}_{Q}\left(x_{p} / y_{q}\right)
$$


and

$$
\left\langle\Omega\left|\mathcal{T}_{Q}\left(x_{q}, y_{q}\right)\right| \bar{\Omega}\right\rangle=N^{1-\frac{1}{2} L} \delta_{Q, 0} \omega^{L(L+1) / 2}\left(y_{p}^{N}-x_{q}^{N}\right)^{r} .
$$

Since $L$ is a multiple of $N$, the right-hand sides of (40) and (42) are equal; this means that the signs of $\mathbf{E}_{j}^{+}$and $\mathbf{E}_{j}^{-}$are equal, and that the transfer matrix can only have the following form for the $2^{r}$-dimensional ground-state sector:

$$
\mathcal{T}_{0}\left(x_{q}, y_{q}\right)=\prod_{j=1}^{r}\left[X_{j}-\mathbf{H}_{j} Y_{j}+\left(\mathbf{E}_{j}^{+}+\mathbf{E}_{j}^{-}\right) Z_{j}\right]
$$

To evaluate $X_{j}, Y_{j}$ and $Z_{j}$, we need to evaluate $\langle\Omega| \mathbf{E}_{m}^{-}$and $\langle\bar{\Omega}| \mathbf{E}_{m}^{+}$. To do so, we invert both (I.42) and

$$
\langle\Omega|\left(\mathbf{x}_{0}^{+}\right)^{(n)}\left(\mathbf{x}_{1}^{-}\right)^{(n-1)}=\sum_{j=1}^{n} \Lambda_{n-j}\langle\Omega| \mathbf{x}_{j-1}^{+}, \quad\langle\bar{\Omega}|\left(\mathbf{x}_{1}^{-}\right)^{(n)}\left(\mathbf{x}_{0}^{+}\right)^{(n-1)}=\sum_{j=1}^{n} \Lambda_{n-j}\langle\bar{\Omega}| \mathbf{x}_{j}^{-},
$$

which can be derived similarly, as

$$
\begin{array}{ll}
\mathbf{x}_{j}^{-}|\Omega\rangle=\sum_{n=1}^{j} S_{j-n}\left(\mathbf{x}_{0}^{+}\right)^{(n-1)}\left(\mathbf{x}_{1}^{-}\right)^{(n)}|\Omega\rangle, & \mathbf{x}_{j-1}^{+}|\bar{\Omega}\rangle=\sum_{n=1}^{j} S_{j-n}\left(\mathbf{x}_{1}^{-}\right)^{(n-1)}\left(\mathbf{x}_{0}^{+}\right)^{(n)}|\bar{\Omega}\rangle, \\
\langle\Omega| \mathbf{x}_{j-1}^{+}=\sum_{n=1}^{j} S_{j-n}\langle\Omega|\left(\mathbf{x}_{0}^{+}\right)^{(n)}\left(\mathbf{x}_{1}^{-}\right)^{(n-1)}, & \langle\bar{\Omega}| \mathbf{x}_{j}^{-}=\sum_{n=1}^{j} S_{j-n}\langle\bar{\Omega}|\left(\mathbf{x}_{1}^{-}\right)^{(n)}\left(\mathbf{x}_{0}^{+}\right)^{(n-1)},
\end{array}
$$

where

$$
\sum_{n=0}^{m} \Lambda_{m-n} S_{n}=\delta_{m, 0}, \quad \text { with } \quad S_{0}=1, \quad \Lambda_{0}=1
$$

This is most easily seen by viewing $\Lambda$ as a lower triangular $r+1$ by $r+1$ matrix with elements $\Lambda_{i, j}=\Lambda_{i-j}$, zero whenever $j>i$, and a similar form for its inverse. Letting

$$
P(z)=\sum_{n=0}^{r} \Lambda_{n} z^{n}, \quad Q(z)=\sum_{n=0}^{r} S_{n} z^{n}
$$

we find from (47) that

$$
Q(z)=1 / P(z)=\prod_{n=1}^{r}\left(z-z_{n}\right)^{-1}=\sum_{i=1}^{r}\left(z-z_{i}\right)^{-1} \prod_{\ell \neq i}^{r}\left(z_{i}-z_{\ell}\right)^{-1} .
$$

Using (10) with $z=0$ together with $\prod_{\ell=1}^{r}\left(-z_{\ell}\right)=1$, we obtain

$$
S_{n}=\sum_{i=1}^{r} z_{i}^{-n} \beta_{i, 0}, \quad \text { for } \quad 1-r<n<r-1,
$$

also using (11), which implies $S_{n}=0$ for $1-r<n<0$. Substituting (46) into (13) with $\ell=0$, interchanging the order of summation, and using (50), we get

$$
\langle\Omega| \mathbf{E}_{m}^{-}=-\langle\Omega| \sum_{\ell=1}^{r}\left(\mathbf{x}_{0}^{+}\right)^{(\ell)}\left(\mathbf{x}_{1}^{-}\right)^{(\ell-1)} \sum_{i=1}^{r} \beta_{i, 0} \sum_{j=0}^{r-1} \beta_{m^{*}, j} z_{i}^{\ell-j-1},
$$

6 
where the condition $S_{n}=0$ for negative values of $n$ has been used to extend the interval of the $j$ summation. As (I.41) implies the symmetry $P(z)=z^{r} P(1 / z)$, we find that for each root $z_{i}$ of $P(z), z_{i^{*}} \equiv 1 / z_{i}$ is also a root. We now use the first equation in (11), i.e.

$$
\sum_{j=0}^{r-1} \beta_{m^{*}, j} z_{i}^{-j}=\sum_{j=0}^{r-1} \beta_{m^{*}, j} z_{i^{*}}^{j}=\delta_{m, i}
$$

to get

$$
\begin{aligned}
& \langle\Omega| \mathbf{E}_{m}^{-}=-\beta_{m, 0} \sum_{\ell=1}^{r} z_{m}^{\ell-1}\langle\Omega|\left(\mathbf{x}_{0}^{+}\right)^{(\ell)}\left(\mathbf{x}_{1}^{-}\right)^{(\ell-1)}, \\
& \mathbf{E}_{m}^{-}|\bar{\Omega}\rangle=-\beta_{m, 0} \sum_{\ell=1}^{r} z_{m}^{\ell-1}\left(\mathbf{x}_{1}^{-}\right)^{(\ell-1)}\left(\mathbf{x}_{0}^{+}\right)^{(\ell)}|\bar{\Omega}\rangle .
\end{aligned}
$$

Similarly, but now using (13) with $\ell=1$, we can show

$$
\begin{aligned}
& \mathbf{E}_{m}^{+}|\Omega\rangle=\beta_{m, 0} \sum_{\ell=1}^{r} z_{m}^{\ell}\left(\mathbf{x}_{0}^{+}\right)^{(\ell-1)}\left(\mathbf{x}_{1}^{-}\right)^{(\ell)}|\Omega\rangle, \\
& \langle\bar{\Omega}| \mathbf{E}_{m}^{+}=\beta_{m, 0} \sum_{\ell=1}^{r} z_{m}^{\ell}\langle\bar{\Omega}|\left(\mathbf{x}_{1}^{-}\right)^{(\ell)}\left(\mathbf{x}_{0}^{+}\right)^{(\ell-1)} .
\end{aligned}
$$

From (I.20), we find ${ }^{7}$

$$
\begin{array}{ll}
\frac{\mathfrak{e}^{n}}{[n] !}\left|n^{\prime}\right\rangle=\left[\begin{array}{c}
n^{\prime} \\
n
\end{array}\right]\left|n^{\prime}-n\right\rangle, & \frac{\mathfrak{f}^{n}}{[n] !}\left|n^{\prime}\right\rangle=\left[\begin{array}{c}
n^{\prime}+n \\
n
\end{array}\right]\left|n^{\prime}+n\right\rangle, \\
\left\langle n^{\prime}\right| \frac{\mathfrak{e}^{n}}{[n] !}=\left[\begin{array}{c}
n^{\prime}+n \\
n
\end{array}\right]\left\langle n^{\prime}+n\right|, & \left\langle n^{\prime}\right| \frac{\mathfrak{f}^{n}}{[n] !}=\left[\begin{array}{c}
n^{\prime} \\
n
\end{array}\right]\left\langle n^{\prime}-n\right| .
\end{array}
$$

Using (I.35) ${ }^{8}$ and the above formulas, we obtain

$$
\begin{aligned}
& \left(\mathbf{x}_{1}^{-}\right)^{(\ell)}|\Omega\rangle=\sum_{\substack{\left\{0 \leqslant n_{j} \leqslant N-1\right\} \\
n_{1}+\cdots+n_{L}=\ell N}} \omega^{-\sum_{j} j n_{j}}\left|\left\{n_{j}\right\}\right\rangle, \\
& \left(\mathbf{x}_{0}^{+}\right)^{(\ell)}|\bar{\Omega}\rangle=(-1)^{\ell} \sum_{\substack{\left\{0 \leqslant n_{j} \leqslant N-1\right\} \\
n_{1}+\cdots+n_{L}=\ell N}}\left|\left\{N-1-n_{j}\right\}\right\rangle, \\
& \langle\Omega|\left(\mathbf{x}_{0}^{+}\right)^{(\ell)}=\sum_{\substack{\left\{0 \leqslant n_{j} \leqslant N-1\right\} \\
n_{1}+\cdots+n_{L}=\ell N}} \omega^{\sum_{j} j n_{j}}\left\langle\left\{n_{j}\right\}\right|, \\
& \langle\bar{\Omega}|\left(\mathbf{x}_{1}^{-}\right)^{(\ell)}=(-1)^{\ell} \sum_{\substack{\left\{0 \leqslant n_{j} \leqslant N-1\right\} \\
n_{1}+\cdots+n_{L}=\ell N}}\left\langle\left\{N-1-n_{j}\right\}\right|,
\end{aligned}
$$

where we have twice used

$$
\left[\begin{array}{c}
N-1-n \\
v
\end{array}\right]=(-1)^{v} \omega^{-n v-v(v+1) / 2}\left[\begin{array}{c}
v+n \\
v
\end{array}\right]
$$

with $n=0$. Continuing the same process we find

$$
\left(\mathbf{x}_{0}^{+}\right)^{(\ell)}\left(\mathbf{x}_{1}^{-}\right)^{(\ell+1)}|\Omega\rangle=\sum_{\substack{\left\{0 \leqslant n_{j} \leqslant N-1\right\} \\ n_{1}+\cdots+n_{L}=N}} \omega^{-\sum_{j} j n_{j}} K_{\ell N}\left(\left\{n_{j}\right\}\right)\left|\left\{n_{j}\right\}\right\rangle,
$$

7 We use the standard definition $\left[\begin{array}{c}n^{\prime} \\ n\end{array}\right] \equiv \frac{\left[n^{\prime}\right] !}{\left.[n] ! n^{\prime}-n\right] !}$.

8 There are some misprints in (I.35): The four subscripts $j$ must be replaced by $m$. 


$$
\begin{aligned}
\langle\Omega|\left(\mathbf{x}_{0}^{+}\right)^{(\ell+1)}\left(\mathbf{x}_{1}^{-}\right)^{(\ell)} & =\sum_{\substack{\left\{0 \leqslant n_{j} \leqslant N-1\right\} \\
n_{1}+\cdots+n_{L}=N}}\left\langle\left\{n_{j}\right\}\right| \omega^{\sum_{j} j n_{j}} \bar{K}_{\ell N}\left(\left\{n_{j}\right\}\right), \\
\left(\mathbf{x}_{1}^{-}\right)^{(\ell)}\left(\mathbf{x}_{0}^{+}\right)^{(\ell+1)}|\bar{\Omega}\rangle & =-\sum_{\substack{\left\{0 \leqslant n_{j} \leqslant N-1\right\} \\
n_{1}+\cdots+n_{L}=N}} K_{\ell N}\left(\left\{n_{j}\right\}\right)\left|\left\{N-1-n_{j}\right\}\right\rangle, \\
\langle\bar{\Omega}|\left(\mathbf{x}_{1}^{-}\right)^{(\ell+1)}\left(\mathbf{x}_{0}^{+}\right)^{(\ell)} & =-\sum_{\substack{\left\{0 \leqslant n_{j} \leqslant N-1\right\} \\
n_{1}+\cdots+n_{L}=N}}\left\langle\left\{N-1-n_{j}\right\}\right| \bar{K}_{\ell N}\left(\left\{n_{j}\right\}\right),
\end{aligned}
$$

where

$$
\begin{array}{ll}
K_{m}\left(\left\{n_{j}\right\}\right) \equiv \sum_{\substack{\left\{0 \leqslant n_{j}^{\prime} \leqslant N-1\right\} \\
n_{1}^{\prime}+\cdots+n_{L}^{\prime}=m}} \prod_{j=1}^{L}\left[\begin{array}{c}
n_{j}+n_{j}^{\prime} \\
n_{j}^{\prime}
\end{array}\right] \omega^{n_{j}^{\prime} N_{j},} & N_{j} \equiv \sum_{\ell=1}^{j-1} n_{\ell}, \\
\bar{K}_{m}\left(\left\{n_{j}\right\}\right) \equiv \sum_{\substack{\left\{0 \leqslant n_{j}^{\prime} \leqslant N-1\right\} \\
n_{1}^{\prime}+\cdots+n_{L}^{\prime}=m}} \prod_{j=1}^{L}\left[\begin{array}{c}
n_{j}+n_{j}^{\prime} \\
n_{j}^{\prime}
\end{array}\right] \omega^{n_{j}^{\prime} \bar{N}_{j},} \quad \bar{N}_{j} \equiv \sum_{\ell=j+1}^{L} n_{\ell},
\end{array}
$$

for integers $m \leqslant(N-1) L$. Also we have $\sum_{j} n_{j}^{\prime} N_{j}=\sum_{j} n_{j} \bar{N}_{j}^{\prime}, \sum_{j} n_{j}^{\prime} \bar{N}_{j}=\sum_{j} n_{j} N_{j}^{\prime}$. The generating function of $K_{m}\left(\left\{n_{j}\right\}\right)$ for $n_{1}+\cdots+n_{L}=k N$ is

$$
g\left(\left\{n_{j}\right\}, t\right) \equiv \sum_{m=0}^{(N-1) L-k N} K_{m}\left(\left\{n_{j}\right\}\right) t^{m}=\frac{1}{\left(1-t^{N}\right)^{k}} \prod_{j=1}^{L} \frac{1-t^{N}}{1-t \omega^{N_{j}}},
$$

which is obtained by inserting $t^{n_{j}^{\prime}}$ in each of the $L$ sums of $K_{m}\left(\left\{n_{j}\right\}\right)$ and summing over $m$ to remove the constraint; after using (58) and other $\omega$-binomial identities we arrive at (62). Similarly, we can define $\bar{g}\left(\left\{n_{j}\right\}, t\right)$ with $K_{m}\left(\left\{n_{j}\right\}\right)$ replaced by $\bar{K}_{m}\left(\left\{n_{j}\right\}\right)$ and $N_{j}$ by $\bar{N}_{j}$. As seen from (60) and (61), here we only need to consider the case with $k=1$.

We define the following polynomials for $n_{1}+\cdots+n_{L}=N$.

$G_{Q}\left(\left\{n_{j}\right\}, z\right) \equiv \sum_{\ell=0}^{m_{Q}-1} K_{Q+\ell N}\left(\left\{n_{i}\right\}\right) z^{\ell}=\frac{t^{-Q}}{N\left(1-t^{N}\right)} \sum_{a=0}^{N-1} \omega^{-Q a} \prod_{j=1}^{L} \frac{1-t^{N}}{1-t \omega^{a+N_{j}}}$,

and

$\bar{G}_{Q}\left(\left\{n_{j}\right\}, z\right) \equiv \sum_{\ell=0}^{m_{Q}-1} \bar{K}_{Q+\ell N}\left(\left\{n_{j}\right\}\right) z^{\ell}=\frac{t^{-Q}}{N\left(1-t^{N}\right)} \sum_{a=0}^{N-1} \omega^{-Q a} \prod_{j=1}^{L} \frac{1-t^{N}}{1-t \omega^{a+\bar{N}_{j}}}$,

where $z=t^{N}$. The last equalities in (63) and (64) are proved noting

$$
G_{Q}\left(\left\{n_{j}\right\}, z\right)=N^{-1} \sum_{a=0}^{N-1}\left(t \omega^{a}\right)^{-Q} g\left(\left\{n_{j}\right\}, t \omega^{a}\right)
$$

and a similar equation for $\bar{G}_{Q}\left(\left\{n_{j}\right\}, z\right)$. For $Q=0$, we drop the subscripts in $G_{0}\left(\left\{n_{j}\right\}, z\right)$ and $\bar{G}_{0}\left(\left\{n_{j}\right\}, z\right)$. It is easy to see that $\bar{G}\left(\left\{n_{j}\right\}, z\right)$ is the complex conjugate of $G\left(\left\{n_{j}\right\}, z\right)$ when $t$ is real.

Substituting (59) into (53) and (54), and using (63) and (64), we obtain

$$
\langle\Omega| \mathbf{E}_{m}^{-}=-\beta_{m, 0} \sum_{\substack{\left\{0 \leqslant n_{j} \leqslant N-1\right\} \\ n_{1}+\cdots+n_{L}=N}}\left\langle\left\{n_{j}\right\}\right| \omega^{\sum_{j} j n_{j}} \bar{G}\left(\left\{n_{j}\right\}, z_{m}\right),
$$




$$
\begin{aligned}
\langle\bar{\Omega}| \mathbf{E}_{m}^{+} & =-\beta_{m, 0} z_{m} \sum_{\substack{\left\{0 \leqslant n_{j} \leqslant N-1\right\} \\
n_{1}+\cdots+n_{L}=N}}\left\langle\left\{N-1-n_{j}\right\}\right| \bar{G}\left(\left\{n_{j}\right\}, z_{m}\right), \\
\mathbf{E}_{k}^{+}|\Omega\rangle & =\beta_{k, 0} z_{k} \sum_{\substack{\left\{0 \leqslant n_{j} \leqslant N-1\right\} \\
n_{1}+\cdots+n_{L}=N}} \omega^{-\sum_{j} j n_{j}} G\left(\left\{n_{j}\right\}, z_{k}\right)\left|\left\{n_{j}\right\}\right\rangle, \\
\mathbf{E}_{k}^{-}|\bar{\Omega}\rangle & =\beta_{k, 0} \sum_{\substack{\left\{0 \leqslant n_{j} \leqslant N-1\right\} \\
n_{1}+\cdots+n_{L}=N}} G\left(\left\{n_{j}\right\}, z_{k}\right)\left|\left\{N-1-n_{j}\right\}\right\rangle .
\end{aligned}
$$

Since

$$
\left\langle\Omega\left|\mathbf{E}_{m}^{-} \mathbf{E}_{k}^{+}\right| \Omega\right\rangle=\delta_{m, k},
$$

we conclude that

$$
\beta_{m, 0} \beta_{k, 0} z_{k} \sum_{\substack{\left\{0 \leqslant n_{j} \leqslant N-1\right\} \\ n_{1}+\cdots+n_{L}=N}} \bar{G}\left(\left\{n_{j}\right\}, z_{m}\right) G\left(\left\{n_{j}\right\}, z_{k}\right)=-\delta_{m k} .
$$

Next, we introduce the polynomials

$$
\mathrm{h}_{k}(z) \equiv \sum_{\substack{\left\{0 \leqslant n_{j} \leqslant N-1\right\} \\ n_{1}+\cdots+n_{L}=N}} \bar{G}\left(\left\{n_{j}\right\}, z_{k}\right) G\left(\left\{n_{j}\right\}, z\right) .
$$

Since the degree of polynomial $G\left(\left\{n_{j}\right\}, z\right)$ defined in (63) is $r-1$, the degree of $\mathrm{h}_{k}(z)$ is also $r-1$. From (71) we know its $r-1$ roots; thus $\mathrm{h}_{k}(z)$ is proportional to $f_{k}(z)$ defined in (10). In fact, we have

$$
\mathrm{h}_{k}(z)=-f_{k}(z) / z_{k} \beta_{k, 0}^{2}=\beta_{k, 0}^{-1} \prod_{\ell \neq k}\left(z-z_{\ell}\right),
$$

where we have used $f_{k}(0)=\beta_{k, 0}$ and $\prod_{\ell=1}^{r}\left(-z_{\ell}\right)=1$. Similarly, we have

$$
\overline{\mathrm{h}}_{k}(z) \equiv \sum_{\substack{\left\{0 \leqslant n_{j} \leqslant N-1\right\} \\ n_{1}+\cdots+n_{L}=N}} \bar{G}\left(\left\{n_{j}\right\}, z\right) G\left(\left\{n_{j}\right\}, z_{k}\right)=\beta_{k, 0}^{-1} \prod_{\ell \neq k}\left(z-z_{\ell}\right) .
$$

We now know enough to calculate the matrix elements we need. Using (66) we find

$$
\left\langle\Omega\left|\mathbf{E}_{m}^{-} \mathcal{T}_{0}\left(x_{q}, y_{q}\right)\right| \Omega\right\rangle=-\beta_{m, 0} \sum_{\substack{\left\{0 \leqslant n_{m} \leqslant N-1\right\} \\ n_{1}+\cdots+n_{L}=N}} \omega^{\sum_{j} j n_{j}} \bar{G}\left(\left\{n_{j}\right\}, z_{m}\right)\left\langle\left\{n_{j}\right\}\left|\mathcal{T}_{0}\left(x_{q}, y_{q}\right)\right| \Omega\right\rangle .
$$

Comparing (37) with (63) for $Q=0$ and using $\sum_{j} N_{j}=\sum_{j}(L-j) n_{j}$, we find

$$
\begin{aligned}
\left\langle\left\{n_{i}\right\}\left|\mathcal{T}_{0}\left(x_{q}, y_{q}\right)\right| \Omega\right\rangle & =N^{-\frac{1}{2} L} \omega^{-\sum_{j} j n_{j}} \sum_{a=0}^{N-1} \prod_{j=1}^{L} \frac{y_{p}^{N}-x_{q}^{N}}{y_{p}-x_{q} \omega^{a+N_{j}}} \\
& =N^{1-\frac{1}{2} L} \omega^{-\sum_{j} j n_{j}} y_{p}^{r N}\left(1-x_{q}^{N} / y_{p}^{N}\right) G\left(\left\{n_{i}\right\},\left(x_{q} / y_{p}\right)^{N}\right) .
\end{aligned}
$$

Using (72), (73) and (76), (75) becomes

$$
\begin{aligned}
\left\langle\Omega\left|\mathbf{E}_{m}^{-} \mathcal{T}_{0}\left(x_{q}, y_{q}\right)\right| \Omega\right\rangle & =-\beta_{m, 0} y_{p}^{r N}\left(1-x_{q}^{N} / y_{p}^{N}\right) N^{1-\frac{1}{2} L} \mathrm{~h}_{m}\left(x_{q}^{N} / y_{p}^{N}\right) \\
& =-y_{p}^{r N}\left(1-x_{q}^{N} / y_{p}^{N}\right) N^{1-\frac{1}{2} L} \prod_{\ell \neq m}\left(x_{q}^{N} / y_{p}^{N}-z_{\ell}\right) .
\end{aligned}
$$


From (43), we find

$$
\left\langle\Omega\left|\mathcal{T}_{0}\left(x_{q}, y_{q}\right)\right| \Omega\right\rangle=\prod_{j=1}^{r}\left(X_{j}+Y_{j}\right), \quad\left\langle\Omega\left|\mathbf{E}_{m}^{-} \mathcal{T}_{0}\left(x_{q}, y_{q}\right)\right| \Omega\right\rangle=Z_{m} \prod_{j \neq m}\left(X_{j}+Y_{j}\right) .
$$

Now equating the ratios given by (78), (39) with (I.44), and (77) we obtain

$$
\frac{X_{m}+Y_{m}}{Z_{m}}=\frac{x_{q}^{N}-y_{p}^{N} z_{m}}{x_{q}^{N}-y_{p}^{N}}
$$

Similarly, we use first (67) and (38), followed by (63), (72) and (73), to find

$$
\begin{aligned}
&\left\langle\bar{\Omega}\left|\mathbf{E}_{m}^{+} \mathcal{T}_{0}\left(x_{q}, y_{q}\right)\right| \bar{\Omega}\right\rangle=-\beta_{m, 0} z_{m}\left(\mu_{p} x_{p} / \mu_{q}\right)^{r N} N^{-\frac{1}{2} L} \\
& \times \sum_{\substack{\left\{0 \leqslant n_{j} \leqslant N-1\right\} \\
n_{1}+\cdots+n_{L}=N}} \bar{G}\left(\left\{n_{j}\right\}, z_{m}\right) \sum_{a=0}^{N-1} \prod_{j=1}^{L} \frac{1-\left(y_{q} / x_{p}\right)^{N}}{1-\omega^{-a-j-N_{j}^{\prime}} y_{q} / x_{p}} \\
&=-z_{m}\left(\mu_{p} x_{p} / \mu_{q}\right)^{r N}\left(1-y_{q}^{N} / x_{p}^{N}\right) N^{1-\frac{1}{2} L} \prod_{\ell \neq m}\left(y_{q}^{N} / x_{p}^{N}-z_{\ell}\right),
\end{aligned}
$$

where $N_{j}^{\prime} \equiv \sum_{\ell=1}^{j-1}\left(N-1-n_{\ell}\right)=N(j-1)+1-j-N_{j}$. Using

$$
\left\langle\bar{\Omega}\left|=(-1)^{r}\left\langle\Omega\left|\left(\mathbf{x}_{0}^{+}\right)^{(r)}=\left\langle\Omega\left|\prod_{j=1}^{r} \mathbf{E}_{j}^{-}, \quad\right| \bar{\Omega}\right\rangle=\left(\mathbf{x}_{0}^{-}\right)^{(r)}\right| \Omega\right\rangle=\prod_{j=1}^{r} \mathbf{E}_{j}^{+}\right| \Omega\right\rangle,
$$

cf (I.35) and (17), we obtain from (43)

$$
\left\langle\bar{\Omega}\left|\mathcal{T}_{0}\left(x_{q}, y_{q}\right)\right| \bar{\Omega}\right\rangle=\prod_{j=1}^{r}\left(X_{j}-Y_{j}\right), \quad\left\langle\bar{\Omega}\left|\mathbf{E}_{m}^{+} \mathcal{T}_{0}\left(x_{q}, y_{q}\right)\right| \bar{\Omega}\right\rangle=Z_{m} \prod_{j \neq m}\left(X_{j}-Y_{j}\right) .
$$

Hence, combining this with (41), (I.44) and (80), one finds

$$
\frac{X_{m}-Y_{m}}{Z_{m}}=\frac{x_{p}^{N}-y_{q}^{N} z_{m}^{-1}}{x_{p}^{N}-y_{q}^{N}} \text {. }
$$

This shows that the rotations $\mathcal{R}$ and $\mathcal{S}$ in (26) can be written as direct products of $r$ two by two matrices $\mathcal{R}_{j}$ and $\mathcal{S}_{j}$,

$$
\mathcal{S}=\prod_{j=1}^{r} \mathcal{S}_{j}, \quad \mathcal{R}=\prod_{j=1}^{r} \mathcal{R}_{j}, \quad \mathcal{S}_{j}\left(A_{j}-\mathbf{H}_{j} B_{j}\right) \mathcal{R}_{j}^{-1}=X_{j}-\mathbf{H}_{j} Y_{j}+\left(\mathbf{E}_{j}^{+}+\mathbf{E}_{j}^{-}\right) Z_{j}
$$

Since $\mathcal{R}$ and $\mathcal{S}$ must be independent of $\lambda_{q}$, the left-hand side of the last equation above is linear in $\lambda_{q}^{-1}$, as seen from (9), and so must its right-hand side be. Thus, the ratios given in (79), (83) and (I.2) yield the following:

$$
\begin{aligned}
& X_{j}+Y_{j}=\epsilon_{j} k\left(y_{p}^{N} z_{j}-x_{q}^{N}\right)=\epsilon_{j}\left[\left(1-k^{\prime} \lambda_{p}\right) z_{j}-\left(1-k^{\prime} \lambda_{q}^{-1}\right)\right], \\
& Z_{j}=\epsilon_{j} k\left(y_{p}^{N}-x_{q}^{N}\right)=\epsilon_{j} \lambda_{p} \lambda_{q}^{-1} k\left(y_{q}^{N}-x_{p}^{N}\right)=\epsilon_{j} k^{\prime}\left(\lambda_{q}^{-1}-\lambda_{p}\right), \\
& X_{j}-Y_{j}=\epsilon_{j} \lambda_{p} \lambda_{q}^{-1} k\left(y_{q}^{N} z_{j}^{-1}-x_{p}^{N}\right)=\epsilon_{j}\left[-k^{\prime} \lambda_{p} z_{j}^{-1}+\lambda_{q}^{-1}\left(k^{\prime}+\lambda_{p} z_{j}^{-1}-\lambda_{p}\right)\right] .
\end{aligned}
$$

We have inserted factors $\lambda_{p} \lambda_{q}^{-1}$ in both the numerator and the denominator of the second ratio in (83) so that they become linear in $\lambda_{q}^{-1}$. Since $\operatorname{det}\left(\mathcal{R}_{j}\right)=1$ and $\operatorname{det}\left(\mathcal{S}_{j}\right)=1$, the determinant is invariant under the transformation in (84), namely

$$
X_{j}^{2}-Y_{j}^{2}-Z_{j}^{2}=A_{j}^{2}-B_{j}^{2}
$$

10 
Using (9), (6) and (5) in this order, we find

$$
A_{j}^{2}-B_{j}^{2}=\rho^{2} k^{2}\left(t_{p}^{N} z_{j}-t_{q}^{N}\right) /\left(k^{\prime} \lambda_{q}\right)
$$

whereas from equations on the left of (85) and (I.5), we obtain

$$
X_{j}^{2}-Y_{j}^{2}-Z_{j}^{2}=\epsilon_{j}^{2} k^{2} \lambda_{q}^{-1} \lambda_{p}\left(z_{j}^{-1}-1\right)\left(t_{p}^{N} z_{j}-t_{q}^{N}\right) .
$$

Consequently we find the multiplicative constants

$\epsilon_{j}^{2}=\bar{\epsilon}_{j}^{2} \rho^{2}=\rho^{2} /\left[k^{\prime}\left(z_{j}^{-1}-1\right) \lambda_{p}\right], \quad \rho^{2}=N^{1 / r} k^{\prime} / k^{2}$.

Since the right-hand side of (84) is explicitly given in (85) and the $\lambda_{q}$ dependence in the left-hand side comes only from $A_{j}$ and $B_{j}$ in (9), we conclude that both sides are linear in $\lambda_{q}^{-1}$. Therefore, we can get two equations by equating the coefficients of the constant and linear terms of (84) separately, i.e.

$\mathcal{S}_{j}\left(\cosh \theta_{j} \mathbf{1}-\sinh \theta_{j} \mathbf{H}_{j}\right) \mathcal{R}_{j}^{-1}=\mathbf{M}, \quad \mathcal{S}_{j}\left(\cosh \theta_{j} \mathbf{1}+\sinh \theta_{j} \mathbf{H}_{j}\right) \mathcal{R}_{j}^{-1}=-\mathbf{N}$,

where the matrices $\mathbf{M}$ and $\mathbf{N}$ depend on $j$ and have elements

$m_{11}=-\bar{\epsilon}_{j} k^{\prime} \lambda_{p} / z_{j}, \quad m_{12}=m_{21}=-\bar{\epsilon}_{j} k^{\prime} \lambda_{p}, \quad m_{22}=\bar{\epsilon}_{j}\left(z_{j}-1-k^{\prime} z_{j} \lambda_{p}\right)$,

$n_{11}=\bar{\epsilon}_{j}\left(z_{j}^{-1} \lambda_{p}-\lambda_{p}+k^{\prime}\right), \quad n_{12}=n_{21}=n_{22}=\bar{\epsilon}_{j} k^{\prime}, \quad k^{\prime}\left(z_{j}^{-1}-1\right) \lambda_{p} \bar{\epsilon}_{j}^{2}=1$.

It is straightforward to solve for $\mathcal{R}_{j}$ and $\mathcal{S}_{j}$. We shall leave the details to the appendix and present the results here. We find that

$$
\mathcal{S}_{j}=\frac{1}{2}\left(s_{11}+s_{22}\right) \mathbf{1}+\frac{1}{2}\left(s_{11}-s_{22}\right) \mathbf{H}_{j}+s_{12} \mathbf{E}_{j}^{+}+s_{21} \mathbf{E}_{j}^{-},
$$

with

$$
\begin{aligned}
& s_{22}=\left(\frac{m_{22} \mathrm{e}^{\theta_{j}}+n_{22} \mathrm{e}^{-\theta_{j}}}{2 \sinh 2 \theta_{j}}\right)^{1 / 2}, \quad s_{12}=\frac{m_{12} \mathrm{e}^{\theta_{j}}+n_{12} \mathrm{e}^{-\theta_{j}}}{m_{22} \mathrm{e}^{\theta_{j}}+n_{22} \mathrm{e}^{-\theta_{j}}} s_{22}, \\
& s_{21}=\frac{\mathrm{e}^{-2 \theta_{j}}-k^{\prime}}{2 s_{12} \sinh 2 \theta_{j}}, \quad s_{11}=\frac{\mathrm{e}^{2 \theta_{j}}-k^{\prime}}{2 s_{22} \sinh 2 \theta_{j}},
\end{aligned}
$$

where the $\theta_{j}$ are defined in (6), while $\mathcal{R}_{j}$ is the transpose of the inverse of $\mathcal{S}_{j}$, that is

$$
r_{22}=s_{11}, \quad r_{21}=-s_{12}, \quad r_{12}=-s_{21}, \quad r_{11}=s_{22} .
$$

We now show that $\mathcal{R}$ and $\mathcal{S}$ in equation (27) for the other two transfer matrices are identical. To calculate the matrix elements of $\hat{\mathcal{T}}_{Q}\left(y_{q}, x_{q}\right)$ we use (2) and (I.4), while using an equation like (34), but with the replacements $p \leftrightarrow p^{\prime}, q \leftrightarrow q^{\prime}$ (or $x_{r} \leftrightarrow y_{r}$ and $\mu_{r} \rightarrow \mu_{r}^{-1}$ with $r=p, q$ ). Similar to (33) and (35), we may then write

$$
\begin{aligned}
\left\langle\left\{n_{i}^{\prime}\right\}\left|\hat{\mathcal{T}}_{Q}\left(y_{q}, x_{q}\right)\right|\left\{n_{i}\right\}\right\rangle=N^{-\frac{1}{2} L} \sum_{a=0}^{N-1} \omega^{-Q a} & \\
\times & \prod_{j=1}^{L}\left[\frac{\left(x_{p}^{N}-y_{q}^{N}\right) \omega^{a-N_{j}+N_{j}^{\prime}}}{x_{p}-y_{q} \omega^{a-N_{j}+N_{j}^{\prime}}}\left(\frac{\mu_{q}}{\mu_{p}}\right)^{n_{j}^{\prime}} \prod_{\ell=1}^{n_{j}^{\prime}} \frac{x_{q}-y_{p} \omega^{\ell+a-N_{j}+N_{j}^{\prime}}}{x_{p}-y_{q} \omega^{\ell+a-N_{j}+N_{j}^{\prime}}}\right] .
\end{aligned}
$$

Here, when $n_{i}=0$ or $n_{i}=N-1$, the above expressions are related to the functions defined in (63) and (64), i.e.

$$
\begin{aligned}
& \left\langle\Omega\left|\hat{\mathcal{T}}_{0}\left(y_{q}, x_{q}\right)\right|\left\{n_{i}\right\}\right\rangle=N^{1-\frac{1}{2} L} \omega^{\sum_{j=1}^{L} j n_{j}} x_{p}^{r N}\left(1-y_{q}^{N} / x_{p}^{N}\right) \bar{G}\left(\left\{n_{i}\right\},\left(y_{q} / x_{p}\right)^{N}\right), \\
& \left\langle\bar{\Omega}\left|\hat{\mathcal{T}}_{0}\left(y_{q}, x_{q}\right)\right|\left\{N-1-n_{i}\right\}\right\rangle=N^{1-\frac{1}{2} L}\left(y_{p} \mu_{q} / \mu_{p}\right)^{r N}\left(1-x_{q}^{N} / y_{p}^{N}\right) \bar{G}\left(\left\{n_{i}\right\},\left(x_{q} / y_{p}\right)^{N}\right) .
\end{aligned}
$$


Combining this with (68), (69) and (74), and also noting that $(1-z) \bar{G}(\{0\}, z)=P(z)$ for $n_{j} \equiv 0$, we can derive

$$
\frac{\left\langle\Omega\left|\hat{\mathcal{T}}_{0}\left(y_{q}, x_{q}\right)\right| \Omega\right\rangle}{\left\langle\Omega\left|\hat{\mathcal{T}}_{0}\left(y_{q}, x_{q}\right) \mathbf{E}_{m}^{+}\right| \Omega\right\rangle}=-\frac{x_{p}^{N}-y_{q}^{N} z_{m}^{-1}}{x_{p}^{N}-y_{q}^{N}}, \quad \frac{\left\langle\bar{\Omega}\left|\hat{\mathcal{T}}_{0}\left(y_{q}, x_{q}\right)\right| \bar{\Omega}\right\rangle}{\left\langle\bar{\Omega}\left|\hat{\mathcal{T}}_{0}\left(y_{q}, x_{q}\right) \mathbf{E}_{m}^{-}\right| \bar{\Omega}\right\rangle}=-\frac{x_{q}^{N}-y_{p}^{N} z_{m}}{x_{q}^{N}-y_{p}^{N}} .
$$

Setting $x_{q} \leftrightarrow y_{q}$ in (98), we find

$$
\frac{\left\langle\Omega\left|\hat{\mathcal{T}}_{0}\left(x_{q}, y_{q}\right)\right| \Omega\right\rangle}{\left\langle\Omega\left|\hat{\mathcal{T}}_{0}\left(x_{q}, y_{q}\right) \mathbf{E}_{m}^{+}\right| \Omega\right\rangle}=-\frac{x_{p}^{N}-x_{q}^{N} z_{m}^{-1}}{x_{p}^{N}-x_{q}^{N}}, \quad \frac{\left\langle\bar{\Omega}\left|\hat{\mathcal{T}}_{0}\left(x_{q}, y_{q}\right)\right| \bar{\Omega}\right\rangle}{\left\langle\bar{\Omega}\left|\hat{\mathcal{T}}_{0}\left(x_{q}, y_{q}\right) \mathbf{E}_{m}^{-}\right| \bar{\Omega}\right\rangle}=-\frac{y_{q}^{N}-y_{p}^{N} z_{m}}{y_{q}^{N}-y_{p}^{N}} .
$$

Similar to (43) and (84), we find that (27) can be written as

$$
\begin{aligned}
& \hat{\mathcal{T}}_{0}\left(y_{q}, x_{q}\right)=\prod_{j=1}^{r}\left[\bar{X}_{j}-\mathbf{H}_{j} \bar{Y}_{j}+\left(\mathbf{E}_{j}^{+}+\mathbf{E}_{j}^{-}\right) \bar{Z}_{j}\right]=\prod_{j=1}^{r} \mathcal{R}_{j}\left(\bar{A}_{j}-\mathbf{H}_{j} \bar{B}_{j}\right) \mathcal{S}_{j}^{-1}, \\
& \hat{\mathcal{T}}_{0}\left(x_{q}, y_{q}\right)=\prod_{j=1}^{r}\left[X_{j}^{\prime}-\mathbf{H}_{j} Y_{j}^{\prime}+\left(\mathbf{E}_{j}^{+}+\mathbf{E}_{j}^{-}\right) Z_{j}^{\prime}\right]=\prod_{j=1}^{r} \mathcal{R}_{j}\left(A_{j}-\mathbf{H}_{j} B_{j}\right) \mathcal{S}_{j}^{-1} .
\end{aligned}
$$

Since $\bar{A}_{j}$ and $\bar{B}_{j}$ are obtained from $A_{j}$ and $B_{j}$ in (9) by letting $\lambda_{q}^{-1} \rightarrow \lambda_{q}$ as follows from (I.2) for $\lambda_{q} \equiv \mu_{q}^{N}$, they are linear in $\lambda_{q}$, so that, similar to (85), (98) yields

$\bar{X}_{j}+\bar{Y}_{j}=\epsilon_{j} \lambda_{p} k\left(x_{p}^{N}-y_{q}^{N} z_{j}^{-1}\right), \quad \bar{Z}_{j}=-\epsilon_{j} \lambda_{p} k\left(x_{p}^{N}-y_{q}^{N}\right)=-\epsilon_{j} \lambda_{q} k\left(x_{q}^{N}-y_{p}^{N}\right)$,

$\bar{X}_{j}-\bar{Y}_{j}=\epsilon_{j} \lambda_{q} k\left(x_{q}^{N}-y_{p}^{N} z_{j}\right)$,

while each term in the product of (101) is linear in $\lambda_{q}^{-1}$, so that (99) leads to

$X_{j}^{\prime}+Y_{j}^{\prime}=\epsilon_{j} \lambda_{p} k\left(x_{p}^{N}-x_{q}^{N} z_{j}^{-1}\right), \quad Z_{j}^{\prime}=-\epsilon_{j} \lambda_{p} k\left(x_{p}^{N}-x_{q}^{N}\right)=-\epsilon_{j} \lambda_{q}^{-1} k\left(y_{q}^{N}-y_{p}^{N}\right)$,

$X_{j}^{\prime}-Y_{j}^{\prime}=\epsilon_{j} \lambda_{q}^{-1} k\left(y_{q}^{N}-y_{p}^{N} z_{j}\right)$.

By equating separately the coefficients of the terms constant and linear in $\lambda_{q}$ of the $j$ th factors of the products on both sides of (100), we find

$\mathcal{R}_{j}\left(\cosh \theta_{j} \mathbf{1}-\sinh \theta_{j} \mathbf{H}_{j}\right) \mathcal{S}_{j}^{-1}=-\mathbf{N}^{-1}, \quad \mathcal{R}_{j}\left(\cosh \theta_{j} \mathbf{1}+\sinh \theta_{j} \mathbf{H}_{j}\right) \mathcal{S}_{j}^{-1}=\mathbf{M}^{-1}$, where the elements of the matrices $\mathbf{M}$ and $\mathbf{N}$ were given in (91) and for their inverses we used $\operatorname{det} \mathbf{M}=\operatorname{det} \mathbf{N}=1$. From (101) we get the same equation (104), as this case differs only by the interchange $q \leftrightarrow q^{\prime}, \lambda_{q} \leftrightarrow 1 / \lambda_{q}$. Taking the inverses of the two equations in (104) we recover (90). This proves that we can indeed use the identical $\mathcal{R}_{j}$ and $\mathcal{S}_{j}$.

To summarize our results, in (43) and (101) we have expressed the transfer matrices of the superintegrable chiral Potts model for the $Q=0$ ground-state sector in terms of the generators of $\mathfrak{s l}_{2}$ algebras, with constants $X_{j}, Y_{j}, Z_{j}$ and $X_{j}^{\prime}, Y_{j}^{\prime}, Z_{j}^{\prime}$ given explicitly in (85) and (103), where $j=1, \ldots, r$ with $r=(N-1) L / N$ and $L$ a multiple of $N$. The corresponding two sets of $2^{r}$ eigenstates for the transfer matrices are given in (28) and (29) with the rotations $\mathcal{R}$ and $\mathcal{S}$ explicitly given in (84) and (92) to (94).

\section{Acknowledgments}

This work was supported in part by the National Science Foundation under grant PHY-0758139 and by the Australian Research Council under Project ID: LX0989627. The authors thank Professor Rodney Baxter for his valuable comments and criticism. The suggestions and interest of Professor Tetsuo Deguchi are also deeply appreciated. The authors also thank their colleagues and the staff at the Centre for Mathematics and its Applications (CMA) and at the Department of Theoretical Physics (RSPE) of Australian National University for their generous support and hospitality. 
Appendix A. $\mathbf{H}_{m}|\Omega\rangle=-|\Omega\rangle$ and $\left(\mathbf{E}_{m}^{+}\right)^{2}|\Omega\rangle=0$

After applying $x_{0}^{+}$to (I.42), we find, using (I.37) and $\mathbf{h}_{n}=x_{0}^{+} x_{n}^{-}-x_{n}^{-} x_{0}^{+}$in (I.34),

$$
n \Lambda_{n}=\sum_{j=1}^{n} d_{j} \Lambda_{n-j}, \quad \text { where } \quad \mathbf{h}_{n}|\Omega\rangle=d_{n}|\Omega\rangle .
$$

Similar to what is done in [7], we multiply both side by $z^{n}$ and then sum over $n$ from 1 to $\infty$. Next, noting $\Lambda_{n}=0$ for $n>r$, we divide the result by $P(z)$ and obtain

$$
z \frac{d}{d z} \ln P(z)=\sum_{n=1}^{\infty} d_{n} z^{n}=\sum_{j=1}^{r} \frac{z}{z-z_{j}}=-\sum_{n=1}^{\infty} z^{n} \sum_{j=1}^{r} z_{j}^{-n} .
$$

This shows $d_{n}=-\sum_{j=1}^{r} z_{j}^{-n}$. Using (11) we obtain

$$
\sum_{n=0}^{r-1} \beta_{m^{*}, n} d_{n+k}=-\sum_{j=1}^{r} \sum_{n=0}^{r-1} \beta_{m^{*}, n} z_{j^{*}}^{n+k}=-\sum_{j=1}^{r} \delta_{m, j} z_{j}^{-k}=-z_{m}^{-k} .
$$

From (13), we find

$$
\mathbf{H}_{m}|\Omega\rangle=\sum_{n=0}^{r-1} \beta_{m^{*}, n} d_{n}|\Omega\rangle=-|\Omega\rangle .
$$

From (15) we see that $\mathbf{E}_{m}^{ \pm}$and $\mathbf{H}_{m}$ generate a finite-dimensional representation of $\mathfrak{s l}(2)$. We also have $\mathbf{E}_{m}^{-}|\Omega\rangle=0$. Thus this representation is spin- $\frac{1}{2}$, proving $\left(\mathbf{E}_{m}^{+}\right)^{2}|\Omega\rangle=0$.

\section{Appendix B. Results for $\mathbf{E}_{j}^{+} \mathbf{E}_{m}^{+}|\Omega\rangle$}

As the identities in (13) hold for the entire eigenspace, whereas the identity in (54) holds only for the ground state, we use (13) with $\ell=0$ for $\mathbf{E}_{j}^{+}$and (54) for $\mathbf{E}_{m}^{+}$to find

$$
\mathbf{E}_{j}^{+} \mathbf{E}_{m}^{+}|\Omega\rangle=\beta_{m, 0} \sum_{\ell=1}^{r} z_{m}^{\ell} \sum_{\ell^{\prime}=0}^{r-1} \beta_{j^{*}, \ell^{\prime}} \mathbf{x}_{\ell^{\prime}}^{-}\left(\mathbf{x}_{0}^{+}\right)^{(\ell-1)}\left(\mathbf{x}_{1}^{-}\right)^{(\ell)}|\Omega\rangle .
$$

As $\left(\mathbf{x}_{m}^{ \pm}\right)^{(n)} \equiv\left(\mathbf{x}_{m}^{ \pm}\right)^{n} / n$ ! for $n \geqslant 0$ and $\left(\mathbf{x}_{m}^{ \pm}\right)^{(n)} \equiv 0$ for $n<0$, it is easy to prove by induction that the following relations hold for the entire eigenspace,

$$
\begin{aligned}
& {\left[\left(\mathbf{x}_{0}^{+}\right)^{(j)}, \mathbf{x}_{k}^{-}\right]=\left(\mathbf{x}_{0}^{+}\right)^{(j-1)} \mathbf{h}_{k}-\mathbf{x}_{k}^{+}\left(\mathbf{x}_{0}^{+}\right)^{(j-2)},} \\
& {\left[\mathbf{x}_{k}^{+},\left(\mathbf{x}_{1}^{-}\right)^{(j)}\right]=\left(\mathbf{x}_{1}^{-}\right)^{(j-1)} \mathbf{h}_{k+1}+\mathbf{x}_{k+2}^{-}\left(\mathbf{x}_{1}^{-}\right)^{(j-2)},} \\
& {\left[\mathbf{h}_{k},\left(\mathbf{x}_{0}^{+}\right)^{(j)}\right]=-2 \mathbf{x}_{k}^{+}\left(\mathbf{x}_{0}^{+}\right)^{(j-1)}, \quad\left[\mathbf{h}_{k},\left(\mathbf{x}_{1}^{-}\right)^{(j)}\right]=2 \mathbf{x}_{k+1}^{-}\left(\mathbf{x}_{0}^{-}\right)^{(j-1)},}
\end{aligned}
$$

which are very similar to those in [7]. Using (B.2) and (A.1) we interchange the order of operations in (B.1), i.e.

$$
\begin{aligned}
\mathbf{x}_{\ell^{\prime}}^{-}\left(\mathbf{x}_{0}^{+}\right)^{(\ell-1)}\left(\mathbf{x}_{1}^{-}\right)^{(\ell)}|\Omega\rangle=\left\{\left(\mathbf{x}_{0}^{+}\right)^{(\ell-1)}\left(\mathbf{x}_{1}^{-}\right)^{(\ell)} \mathbf{x}_{\ell^{\prime}}^{-}-\left(\mathbf{x}_{0}^{+}\right)^{(\ell-2)}\left[2\left(\mathbf{x}_{1}^{-}\right)^{(\ell-1)} \mathbf{x}_{\ell^{\prime}+1}^{-}\right.\right. \\
\left.\left.+\left(\mathbf{x}_{1}^{-}\right)^{(\ell)} d_{\ell^{\prime}}\right]+\left(\mathbf{x}_{0}^{+}\right)^{(\ell-3)}\left[\left(\mathbf{x}_{1}^{-}\right)^{(\ell-2)} \mathbf{x}_{\ell^{\prime}+2}^{-}+\left(\mathbf{x}_{1}^{-}\right)^{(\ell-1)} d_{\ell^{\prime}+1}\right]\right\}|\Omega\rangle .
\end{aligned}
$$

Substituting (B.3) into (B.1), and using (13) and (A.3), we get 


$$
\begin{aligned}
& \mathbf{E}_{j}^{+} \mathbf{E}_{m}^{+}|\Omega\rangle=\beta_{m, 0}\left\{\sum_{\ell=1}^{r} z_{m}^{\ell}\left(\mathbf{x}_{0}^{+}\right)^{(\ell-1)}\left(\mathbf{x}_{1}^{-}\right)^{(\ell)} \mathbf{E}_{j}^{+}|\Omega\rangle\right. \\
&-\sum_{\ell=2}^{r} z_{m}^{\ell}\left(\mathbf{x}_{0}^{+}\right)^{(\ell-2)}\left[\left(\mathbf{x}_{1}^{-}\right)^{(\ell-1)} 2 z_{j}^{-1} \mathbf{E}_{j}^{+}-\left(\mathbf{x}_{1}^{-}\right)^{(\ell)}\right]|\Omega\rangle \\
&\left.+\sum_{\ell=3}^{r} z_{m}^{\ell}\left(\mathbf{x}_{0}^{+}\right)^{(\ell-3)}\left[\left(\mathbf{x}_{1}^{-}\right)^{(\ell-2)} z_{j}^{-2} \mathbf{E}_{j}^{+}-\left(\mathbf{x}_{1}^{-}\right)^{(\ell-1)} z_{j}^{-1}\right]|\Omega\rangle\right\} \\
&=\beta_{m, 0}\left\{\left(1-2 z_{m} / z_{j}+z_{m}^{2} / z_{j}^{2}\right) \sum_{\ell=1}^{r-1} z_{m}^{\ell}\left(\mathbf{x}_{0}^{+}\right)^{(\ell-1)}\left(\mathbf{x}_{1}^{-}\right)^{(\ell)} \mathbf{E}_{j}^{+}|\Omega\rangle\right. \\
&+\left(1-z_{m} / z_{j}\right) \sum_{\ell=2}^{r} z_{m}^{\ell}\left(\mathbf{x}_{0}^{+}\right)^{(\ell-2)}\left(\mathbf{x}_{1}^{-}\right)^{(\ell)}|\Omega\rangle \\
&+z_{m}^{r}\left(\mathbf{x}_{0}^{+}\right)^{(r-1)}\left(\mathbf{x}_{1}^{-}\right)^{(r)} \mathbf{E}_{j}^{+}|\Omega\rangle \\
&\left.-z_{m}^{r+1}\left(\mathbf{x}_{0}^{+}\right)^{(r-2)}\left[z_{j}^{-2}\left(\mathbf{x}_{1}^{-}\right)^{(r-1)} \mathbf{E}_{j}^{+}-\left(\mathbf{x}_{1}^{-}\right)^{(r)} z_{j}^{-1}\right]|\Omega\rangle\right\} .
\end{aligned}
$$

To arrive at the second equality, we changed the summations in the first equality replacing $\ell-1 \rightarrow \ell$ both in the first part of the second sum and in the second part of the third sum, and $\ell-2 \rightarrow \ell$ in the first part of the third sum. If we can use the identities

$$
\left(\mathbf{x}_{1}^{-}\right)^{(r)} \mathbf{E}_{j}^{+}|\Omega\rangle=0, \quad\left(\mathbf{x}_{1}^{-}\right)^{(r-1)} \mathbf{E}_{j}^{+}|\Omega\rangle=z_{j}\left(\mathbf{x}_{1}^{-}\right)^{(r)}|\Omega\rangle
$$

we may simplify (B.4) to

$$
\begin{aligned}
\mathbf{E}_{j}^{+} \mathbf{E}_{m}^{+}|\Omega\rangle=\beta_{m, 0} & \left\{\left(1-z_{m} / z_{j}\right)^{2} \sum_{\ell=1}^{r-1} z_{m}^{\ell}\left(\mathbf{x}_{0}^{+}\right)^{(\ell-1)}\left(\mathbf{x}_{1}^{-}\right)^{(\ell)} \mathbf{E}_{j}^{+}|\Omega\rangle\right. \\
& \left.+\left(1-z_{m} / z_{j}\right) \sum_{\ell=2}^{r} z_{m}^{\ell}\left(\mathbf{x}_{0}^{+}\right)^{(\ell-2)}\left(\mathbf{x}_{1}^{-}\right)^{(\ell)}|\Omega\rangle\right\}
\end{aligned}
$$

This is clearly consistent with $\left(\mathbf{E}_{m}^{+}\right)^{2}|\Omega\rangle=0$.

From (68), (I.35) and (55), it is easy to see that the first identity in (B.5) holds. To prove the second identity, we use (56) with $n_{j} \equiv N-1$ to find

$$
\left(\mathbf{x}_{1}^{-}\right)^{(r)}|\Omega\rangle=\omega^{\frac{1}{2} L(L+1)}|\bar{\Omega}\rangle .
$$

Next use (68), (I.35) with $v_{m}=N-1-n_{m},(55)$, and finally (58) with $n=0$, to find

$$
\left(\mathbf{x}_{1}^{-}\right)^{(r-1)} \mathbf{E}_{j}^{+}|\Omega\rangle=\omega^{\frac{1}{2} L(L+1)}|\bar{\Omega}\rangle\left(-z_{j} \beta_{j, 0}\right) \sum_{\substack{\left\{0 \leqslant n_{m} \leqslant N-1\right\} \\ n_{1}+\cdots+n_{L}=N}} \omega^{-\sum_{m} m n_{m}} G\left(\left\{n_{m}\right\}, z_{j}\right) .
$$

Multiplying both sides of (74) by $z^{-r+1}$ and then taking the limit $z \equiv t^{N} \rightarrow \infty$, using (64) to obtain the limit $t^{-1} \rightarrow 0$ of $t^{-N r+N} \bar{G}$, where $\sum_{m} \bar{N}_{m}=\sum_{m}(m-1) n_{m}$, we find

$$
\lim _{z \rightarrow \infty} z^{-r+1} \overline{\mathrm{h}}_{k}(z)=-\sum_{\substack{\left\{0 \leqslant n_{j} \leqslant N-1\right\} \\ n_{1}+\cdots+n_{L}=N}} \omega^{-\sum_{m} m n_{m}} G\left(\left\{n_{j}\right\}, z_{k}\right)=-\beta_{k, 0}^{-1} .
$$

Substituting this equation into (B.8), we show that the second identity in (B.5) holds. 


\section{Appendix $\mathbf{C}$. The rotations $\mathbf{R}_{j}$ and $\mathbf{S}_{j}$}

We rewrite (90) as

$$
\mathcal{S}_{j}\left(\cosh \theta_{j} \mathbf{1}-\sinh \theta_{j} \mathbf{H}_{j}\right)=\mathbf{M} \mathcal{R}_{j}, \quad \mathcal{S}_{j}\left(\cosh \theta_{j} \mathbf{1}+\sinh \theta_{j} \mathbf{H}_{j}\right)=-\mathbf{N} \mathcal{R}_{j},
$$

where $\operatorname{det}(\mathbf{M})=\operatorname{det}(\mathbf{N})=1$. We multiply the first equation with $\mathbf{M}^{-1}$ and the second equation with $-\mathbf{N}^{-1}$, so that the right-hand sides of both equations become $\mathcal{R}_{j}$ and the left-hand sides must be equal. This way we obtain a matrix equation for $\mathcal{S}_{j}$. More explicitly its elements $s_{i j}$ can be shown to satisfy the following equations:

$$
\begin{aligned}
& s_{21}\left(m_{11} \mathrm{e}^{-\theta_{j}}+n_{11} \mathrm{e}^{\theta_{j}}\right)=s_{11}\left(m_{21} \mathrm{e}^{-\theta_{j}}+n_{21} \mathrm{e}^{\theta_{j}}\right), \\
& s_{21}\left(m_{12} \mathrm{e}^{-\theta_{j}}+n_{12} \mathrm{e}^{\theta_{j}}\right)=s_{11}\left(m_{22} \mathrm{e}^{-\theta_{j}}+n_{22} \mathrm{e}^{\theta_{j}}\right), \\
& s_{22}\left(m_{11} \mathrm{e}^{\theta_{j}}+n_{11} \mathrm{e}^{-\theta_{j}}\right)=s_{12}\left(m_{21} \mathrm{e}^{\theta_{j}}+n_{21} \mathrm{e}^{-\theta_{j}}\right), \\
& s_{22}\left(m_{12} \mathrm{e}^{\theta_{j}}+n_{12} \mathrm{e}^{-\theta_{j}}\right)=s_{12}\left(m_{22} \mathrm{e}^{\theta_{j}}+n_{22} \mathrm{e}^{-\theta_{j}}\right),
\end{aligned}
$$

These equations are consistent if and only if

$$
\left(m_{11} \mathrm{e}^{\mp \theta_{j}}+n_{11} \mathrm{e}^{ \pm \theta_{j}}\right)\left(m_{22} \mathrm{e}^{\mp \theta_{j}}+n_{22} \mathrm{e}^{ \pm \theta_{j}}\right)=\left(m_{21} \mathrm{e}^{\mp \theta_{j}}+n_{21} \mathrm{e}^{ \pm \theta_{j}}\right)\left(m_{12} \mathrm{e}^{\mp \theta_{j}}+n_{12} \mathrm{e}^{ \pm \theta_{j}}\right) .
$$

We can simplify (C.3) using $m_{12}=m_{21}$ and $n_{12}=n_{21}$ found in (91) and again using $\operatorname{det}(\mathbf{M})=\operatorname{det}(\mathbf{N})=1$. We find that the two conditions become

$$
2 \cosh 2 \theta_{j}=2 m_{12} n_{12}-m_{11} n_{22}-m_{22} n_{11} .
$$

Next, using (5) and (6), we can show

$$
\lambda_{p}+\lambda_{p}^{-1}=2 z_{j}^{-1} \cosh 2 \theta_{j}-\left(k^{\prime}+k^{\prime-1}\right)\left(z_{j}^{-1}-1\right) .
$$

With the help of (91) and (C.5) we can then prove that (C.4) holds.

Moreover, from det $\mathcal{S}_{j}=1$ we calculate $s_{11} s_{22}$ eliminating first $s_{12} s_{12}$ using (C.2) and (91), and then substituting $z_{j}$ from (C.5). This way we find

$$
s_{11} s_{22}=\frac{\mathrm{e}^{2 \theta_{j}}-k^{\prime}}{2 \sinh 2 \theta_{j}}, \quad s_{12} s_{21}=\frac{\mathrm{e}^{-2 \theta_{j}}-k^{\prime}}{2 \sinh 2 \theta_{j}} .
$$

Following nearly identical steps, we obtain from (C.1) consistent equations similar to (C.2) and (C.6) for the matrix elements $r_{i j}$ of $\mathcal{R}_{j}$. From these results we conclude

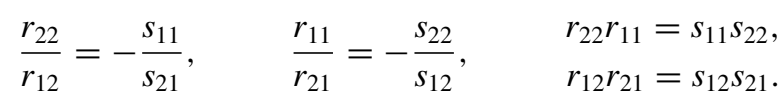

Only six of the equations in (C.2), (C.6) and (C.7) are independent. At this point we may choose $s_{22}$ and $r_{22}$ as free parameters, but then the other six elements are uniquely determined. However, there is one more relation. From direct multiplication of the matrices in (90), we find

$m_{11}=\mathrm{e}^{-\theta_{j}} s_{11} r_{22}-\mathrm{e}^{\theta_{j}} s_{12} r_{21}, \quad n_{11}=-\mathrm{e}^{\theta_{j}} s_{11} r_{22}+\mathrm{e}^{-\theta_{j}} s_{12} r_{21}$.

Eliminating $s_{12} r_{21}$ from these (and similarly starting with $m_{22}$ and $n_{22}$ ), we find

$$
s_{11} r_{22}=-\frac{m_{11} \mathrm{e}^{-\theta_{j}}+n_{11} \mathrm{e}^{\theta_{j}}}{2 \sinh 2 \theta_{j}}, \quad s_{22} r_{11}=\frac{m_{22} \mathrm{e}^{\theta_{j}}+n_{22} \mathrm{e}^{-\theta_{j}}}{2 \sinh 2 \theta_{j}} .
$$

Let us define

$$
T_{i k} \equiv m_{i k} \mathrm{e}^{-\theta_{j}}+n_{i k} \mathrm{e}^{\theta_{j}}=T_{k i}, \quad T_{i k}^{*} \equiv m_{i k} \mathrm{e}^{\theta_{j}}+n_{i k} \mathrm{e}^{-\theta_{j}}=T_{k i}^{*} .
$$


Then it can be easily verified, substituting (91) and eliminating $z_{j}$ from (C.5), that

$$
\begin{aligned}
& T_{12} T_{12}^{*}=-\left(\mathrm{e}^{-2 \theta_{j}}-k^{\prime}\right)\left(\mathrm{e}^{2 \theta_{j}}-k^{\prime}\right), \\
& T_{11} T_{22}^{*}=-\left(\mathrm{e}^{2 \theta_{j}}-k^{\prime}\right)^{2}, \quad T_{22} T_{11}^{*}=-\left(\mathrm{e}^{-2 \theta_{j}}-k^{\prime}\right)^{2} .
\end{aligned}
$$

Using (C.6) and (C.11), we may rewrite (C.9) as

$$
\frac{r_{22}}{s_{22}}=-\frac{T_{11}}{\mathrm{e}^{2 \theta_{j}}-k^{\prime}}=\frac{\mathrm{e}^{2 \theta_{j}}-k^{\prime}}{T_{22}^{*}} .
$$

This shows that there is only one free parameter $s_{22}$, and we choose it so that $r_{11}=s_{22}$. This choice yields the solution given in (93) and (94).

\section{References}

[1] Au-Yang H and Perk J H H 2008 Eigenvectors in the superintegrable model I: $\mathfrak{s l}_{2}$ generators J. Phys. A: Math. Theor. 41275201

[2] Baxter R J 1989 The superintegrable chiral Potts model: thermodynamic properties, an 'inverse model', and a simple associated Hamiltonian J. Stat. Phys. 57 1-39

[3] Baxter R J 1993 Chiral Potts model with skewed boundary conditions J. Stat. Phys. 73 461-95

[4] Baxter R J 1994 Interfacial tension of the chiral Potts model J. Phys. A: Math. Gen. 27 1837-49

[5] Davies B 1990 Onsager's algebra and superintegrability J. Phys. A: Math. Gen. 23 2245-61

[6] Prony (G C F M R de) 1795 Considérations sur les principes de la méthode inverse des différences $J$. de l'Éc. Polyt. 1 (3) 209-73 (see pp 264-5)

[7] Deguchi T 2007 Regular XXZ Bethe states at roots of unity as highest weight vectors of the $s l_{2}$ loop algebra J. Phys. A: Math. Theor. $407473-508$

[8] Nishino A and Deguchi T 2006 The $L\left(\mathfrak{s} l_{2}\right)$ symmetry of the Bazhanov-Stroganov model associated with the superintegrable chiral Potts model Phys. Lett. A 356 366-70

[9] Deguchi T 2007 Irreducibility criterion for a finite-dimensional highest weight representation of the $s l_{2}$ loop algebra and the dimensions of reducible representations J. Stat. Mech. P05007

[10] Nishino A and Deguchi T 2008 An algebraic derivation of the eigenspaces associated with an Ising-like spectrum of the superintegrable chiral Potts model J. Stat. Phys. 133 587-615

[11] Onsager L 1944 Crystal statistics: I. A two-dimensional model with an order-disorder transition Phys. Rev. 65 117-49 